$\xi=\square$

\title{
Non topological 1-soliton solution to resonant nonlinear schrodinger equation with kerr law nonlinearity
}

\author{
S. Subhaschandra Singh * \\ Physics Department, Imphal College, Imphal - 795001,Manipur, India \\ *Corresponding author E-mail: subhasic@yahoo.co.in
}

\begin{abstract}
In this paper, using the methods of ansatz, sine-cosine and He's semi-inverse variation, non-topological 1-soliton solution to Resonant Nonlinear Schrodinger Equation with Kerr law nonlinearity is obtained. The results show that these methods are very effective ones for finding exact solutions to various types of nonlinear evolution equations appearing in the studies of science and engineering.
\end{abstract}

Keywords: Temporal evolution; Group Velocity Dispersion (GVD); Ritz method.

\section{Introduction}

The Resonant Nonlinear Schrodinger Equation $[1,2]$ is encountered in the studies of Nonlinear Fiber Optics, Fluid Physics and Plasma Physics. The dimensionless form of this equation is often written in the form

$i \frac{\partial \Psi}{\partial t}+\alpha \frac{\partial^{2} \Psi}{\partial x^{2}}+\beta F\left(|\Psi|^{2}\right) \Psi+\gamma\left\{\frac{\frac{\partial^{2}|\Psi|}{\partial x^{2}}}{|\Psi|}\right\} \Psi=0$

where the first term represents the temporal evolution term, the second term represents the Group Velocity Dispersion (GVD) term, the third term represents the nonlinearity term and the fourth term represents the resonant nonlinearity term. Moreover, $i=$ $\sqrt{ }(-1)$ is the imaginary number, $\alpha$ is the coefficient of GVD, $\beta$ is the coefficient of nonlinearity, $\gamma$ is the coefficient of resonant nonlinearity and $F(s)$ is a function defining the type of nonlinearity. The dependent variable $\Psi(x, t)$ is a complex valued wave profile and the independent variables $x$ and $t$ represent dimensionless distance in the direction of propagation and the retarded time in the GVD frame. For Kerr law nonlinearity, we have, $F(s)=s$ and, hence, we write equation (1) as

$i \frac{\partial \Psi}{\partial t}+\alpha \frac{\partial^{2} \Psi}{\partial x^{2}}+\beta|\Psi|^{2} \Psi+\gamma\left\{\frac{\frac{\partial^{2}|\Psi|}{\partial x^{2}}}{|\Psi|}\right\} \Psi=0$.

In the present paper, non-topological 1-soliton solution to equation (2) is found out using the methods of ansatz, sine-cosine and He's semi-inverse variation.

\section{Reduction to nonlinear ordinary differential equation}

To reduce Eq. (2) to a nonlinear ordinary differential equation (NLODE), let us put

$\Psi(x, t)=u(\xi) e^{i(-k x+\omega t)}, \xi=x-v t$ where $k$ represents the frequency, $\omega$ represents the propagation number and $v$ represents the propagation speed of the soliton.

Using the above transformations, we obtain

$$
\begin{aligned}
& i \frac{\partial \Psi}{\partial t}=i\left(-v \frac{d u}{d \xi}+i \omega u\right) e^{i(-k x+\omega t)}, \\
& \alpha \frac{\partial^{2} \Psi}{\partial x^{2}}=\alpha\left(\frac{d^{2} u}{d \xi^{2}}-2 i k \frac{d u}{d \xi}-k^{2} u\right) e^{i(-k x+\omega t)} \\
& \beta|\Psi|^{2} \Psi=\beta u^{3} e^{i(-k x+\omega t)}, \\
& \gamma\left\{\frac{\frac{\partial^{2}|\Psi|}{\partial x^{2}}}{|\Psi|}\right\} \Psi=\gamma \frac{d^{2} u}{d \xi^{2}} e^{i(-k x+\omega t)} .
\end{aligned}
$$

Substituting Eqs. (4) into Eq. (2), we obtain

$$
(\alpha+\gamma) \frac{d^{2} u}{d \xi^{2}}-i(v+2 \alpha k) \frac{d u}{d \xi}-\left(\omega+\alpha k^{2}\right) u+\beta u^{3}=0 .
$$

Splitting Eq. (5) into real and imaginary parts, we write

Real Part:

$$
(\alpha+\gamma) \frac{d^{2} u}{d \xi^{2}}-\left(\omega+\alpha k^{2}\right) u+\beta u^{3}=0
$$

Imaginary part:

$$
v=-2 \alpha k \text {. }
$$

Thus, if we solve $u(\xi)$ from Eq. (6), we can find the solution of Eq. (2) using the transformations (3) together with Eq. (7).

\section{Solution via ansatz method}

In the ansatz method [3 - 5] of finding bright or non-topological soliton solution, we assume the solution of Eq. (6) as 
$u(\xi)=A \operatorname{sech}^{p}(B \xi)$

where $A, B, p$ are parameters to be determined latter. The parameter $A$ is called soliton amplitude and the parameter $B$ is called in verse width of soliton.

Substituting Eq. (8) and its second order derivative into Eq. (6), we obtain

$$
\begin{aligned}
& (\alpha+\gamma)\left[p^{2} A B^{2} \operatorname{sech}^{p}(B \xi)\right]-p(p+1) A B^{2} \operatorname{sech}^{p+2}(B \xi) \\
& -\left(\omega+\alpha k^{2}\right) A \operatorname{sech}^{p}(B \xi)+\beta A^{3} \operatorname{sech}^{3 p}(B \xi)=0 .
\end{aligned}
$$

From Eq. (9), equating the exponents $(p+2)$ and $3 p$, we obtain $p=1$.

Again, from Eq. (9), equating the coefficients of $\operatorname{sech}^{3}(B \xi)$ to zero, we obtain

$\beta A^{2}=2(\alpha+\gamma) B^{2}$ with $A \neq 0$.

Further, from Eq. (9), equating the coefficient of $\operatorname{sech}(B \xi)$ to zero, we obtain

$B= \pm \sqrt{\frac{\omega+\alpha k^{2}}{\alpha+\gamma}}$

Substitution of Eq. (11) into Eq. (10) yields

$A= \pm \sqrt{\frac{2\left(\omega+\alpha k^{2}\right)}{\beta}}$

Substituting the values of $A, B$ and $p$ into Eq. (8), we obtain

$u(\xi)= \pm \sqrt{\frac{2\left(\omega+\alpha k^{2}\right)}{\beta}} \operatorname{sech}\left\{\left(\sqrt{\frac{\omega+\alpha k^{2}}{\alpha+\gamma}}\right) \xi\right\}$

Substituting Eq. (13) with the positive coefficient into Eq. (3) and recalling the expressions for $v$ and $\xi$, we obtain

$\Psi(x, t)$

$=\sqrt{\frac{2\left(\omega+\alpha k^{2}\right)}{\beta}}\left[\operatorname{sech}\left\{\left(\sqrt{\frac{\omega+\alpha k^{2}}{\alpha+\gamma}}\right)(x+2 \alpha k t)\right\}\right] e^{i(-k x+\omega t)}$.

Here, Eq. (14) represents Bright or Non-topological 1- soliton solution of Resonant Nonlinear Schrodinger Equation with Kerr law nonlinearity.

\section{Solution via sine-cosine method}

In the sine-cosine method [6 - 10], the solution of Eq. (6) is assumed as in either of the forms

$$
u(\xi)=A \sin ^{\lambda}(\mu \xi)
$$

Or, $u(\xi)=A \cos ^{\lambda}(\mu \xi)$

where in either case, $A, \lambda$ and $\mu$ are parameters to be determined latter.

In the present work, let us choose the cosine form in Eq. (16).

Substituting Eq. (16) and its second order derivative into Eq. (6), we obtain

$$
\begin{aligned}
& (\alpha+\gamma)\left\{A \lambda(\lambda-1) \mu^{2} \cos ^{\lambda-2}(\mu \xi)-A \lambda^{2} \mu^{2} \cos ^{\lambda}(\mu \xi)\right\} \\
& -\left(\omega+\alpha k^{2}\right) A \cos ^{\lambda}(\mu \xi)+\beta A^{3} \cos ^{3 \lambda}(\mu \xi)=0 .
\end{aligned}
$$

From Eq. (17), equating the exponents $(\lambda-2)$ and $3 \lambda$, we obtain $\lambda=-1$.

Again, equating the coefficient of $\cos ^{-3}(\mu \xi)$ to zero, we obtain $2(\alpha+\gamma) \mu^{2}+\beta A^{2}=0$, with $A \neq 0$.
Further, from Eq. (17), equating the coefficient of $\cos ^{-1}(\mu \xi)$ to zero, we obtain

$-(\alpha+\gamma) \mu^{2}-\left(\omega+\alpha k^{2}\right)=0$

yielding

$\mu= \pm i \sqrt{\frac{\omega+\alpha k^{2}}{\alpha+\gamma}}$.

Substituting Eq. (19) into Eq. (18), we obtain

$A= \pm \sqrt{\frac{2\left(\omega+\alpha k^{2}\right)}{\beta}}$

Substituting the values of $A, \mu$ and $\lambda$ into Eq. (16), we obtain

$u(\xi)= \pm \sqrt{\frac{2\left(\omega+\alpha k^{2}\right)}{\beta}} \sec \left\{i\left(\sqrt{\frac{\omega+\alpha k^{2}}{\alpha+\gamma}}\right) \xi\right\}$

$= \pm \sqrt{\frac{2\left(\omega+\alpha k^{2}\right)}{\beta}} \operatorname{sech}\left\{\left(\sqrt{\frac{\omega+\alpha k^{2}}{\alpha+\gamma}}\right) \xi\right\}$

Substituting Eq. (21) with the positive coefficient into Eq. (3) and recalling the expressions for $\xi$ and $v$, we obtain the solution of Eq. (2) as

$\Psi(x, t)$

$=\sqrt{\frac{2\left(\omega+\alpha k^{2}\right)}{\beta}}\left[\operatorname{sech}\left\{\left(\sqrt{\frac{\omega+\alpha k^{2}}{\alpha+\gamma}}\right)(x+2 \alpha k t)\right\}\right] e^{i(-k x+\omega t)}$.

We see that the solution obtained here is just the non-topological 1 -soliton solution obtained earlier by the method of ansatz.

\section{Solution via He's semi-inverse variation method}

In this section, the solution of Eq. (6) is to be obtained via He's semi-inverse variation method [4], [11 -15]. However, before applying the method, let us first introduce a brief description of the method as in the following.

\subsection{Description of He's semi-inverse variation method}

If the nonlinear partial differential equation to be solved is in the form

$P\left(u, u_{t}, u_{x}, u_{t t}, u_{x x}, u_{t x}, \ldots\right)=0$

where $t$ and $x$ are independent variables and the subscripts denote partial derivatives of the dependent variable $u$ with respect to them, and then we are to perform the following operational steps.

Step 1: The given nonlinear partial differential equation (NLPDE) is first to be reduced to a nonlinear ordinary differential equation (NLODE) by introducing a transformation

$u(x, t)=U(\xi)$ and $\xi=x-v t$ as in section 2.

Let us write the reduced NLODE as

$Q\left(U, U^{\prime}, U^{\prime \prime}, \ldots\right)=0$

where $U^{\prime}=\frac{d U}{d \xi}, \quad U^{\prime \prime}=\frac{d^{2} U}{d \xi^{2}}$, etc.

Step 2: The reduced NLODE is to be integrated once or more so long as every term in that equation contains derivative(s) and for 
the sake of simplicity, we are to choose the integration constant(s) as zero.

Step 3: Next, we are to construct a trial function $J(U)=\int_{a}^{b} L d \xi$

where $a$ and $b$ are the lower and the upper limits related to the problem and $L$ is a function of $U$ and its derivatives.

Step 4: In accordance with Ritz method, we assume a solitary wave solution $U$ in any one of the forms

$$
\begin{aligned}
& U(\xi)=A \operatorname{sech}^{p}(B \xi), \quad U(\xi)=A \operatorname{cosech}^{p}(B \xi), \\
& U(\xi)=A \tanh ^{p}(B \xi), \quad U(\xi)=A \operatorname{coth}(B \xi), \text { etc. }
\end{aligned}
$$

Here, the parameter $p$ is to be determined through a balancing of degrees between the term with the highest derivative and the term with highest nonlinearity. The remaining parameters $A$ and $B$ are to be determined by substituting the assumed solitary solution to Eq. (24) and making $J$ stationary with respect to $A$ and $B$, that is making

$$
\frac{\partial J}{\partial A}=0
$$

and $\quad \frac{\partial J}{\partial B}=0$.

Solving Eqs. (25) And (26), we will obtain the values of the parameters $A$ and $B$. Then, substituting the values of $p, A$ and $B$ into the assumed solitary wave solution, we can obtain a solution of the Reduced NLODE and hence of the given NLPDE.

\subsection{Application of semi-inverse variation method}

From Equation (6), balancing of degrees between the term with highest order derivative and the term with highest nonlinearity results in

$$
p+2=3 p \text { giving } p=1 \text {. }
$$

Now, multiplying both sides of Eq. (6) by $\frac{d u}{d \xi}$, we obtain

$$
(\alpha+\gamma) \frac{d u}{d \xi} \frac{d^{2} u}{d \xi^{2}}-\left(\omega+\alpha k^{2}\right) u \frac{d u}{d \xi}+\beta u^{3} \frac{d u}{d \xi}=0
$$

Integrating both sides with respect to $\xi$ and choosing the integration constant as zero, we obtain

$$
\frac{(\alpha+\gamma)}{2}\left(\frac{d u}{d \xi}\right)^{2}-\frac{\left(\omega+\alpha k^{2}\right)}{2} u^{2}+\frac{\beta}{4} u^{4}=0 .
$$

Next, we construct the trial function

$$
\begin{aligned}
J & =J(u) \\
& =\int_{0}^{\infty}\left[-\frac{(\alpha+\gamma)}{2}\left(\frac{d u}{d \xi}\right)^{2}-\frac{\left(\omega+\alpha k^{2}\right)}{2} u^{2}+\frac{\beta}{4} u^{4}\right] d \xi .
\end{aligned}
$$

We assume a solitary wave solution in the form

$$
u(\xi)=A \operatorname{sech}^{p}(B \xi)=A \operatorname{sech}(B \xi) .
$$

Substitution of Eq. (28) into Eq. (27) yields

$$
\begin{aligned}
& J=\int_{0}^{\infty}\left[\begin{array}{c}
-\frac{(\alpha+\gamma)}{2} A^{2} B^{2} \operatorname{sech}^{2}(B \xi) \tanh ^{2}(B \xi) \\
-\frac{\left(\omega+\alpha k^{2}\right)}{2} A^{2} \operatorname{sech}^{2}(B \xi)+\frac{\beta}{4} A^{4} \operatorname{sech}^{4}(B \xi)
\end{array}\right] d \xi \\
& =-\frac{(\alpha+\gamma)}{6} A^{2} B-\frac{\left(\omega+\alpha k^{2}\right)}{2 B} A^{2}+\frac{\beta}{6 B} A^{4} .
\end{aligned}
$$

Making $J$ stationary with respect to $A$ and $B$, we obtain respectively

$$
\frac{\partial J}{\partial A}=-\frac{(\alpha+\gamma)}{3} A B-\left(\omega+\alpha k^{2}\right) \frac{A}{B}+\frac{2 \beta}{3} \frac{A^{3}}{B}=0
$$

and

$$
\frac{\partial J}{\partial B}=-\frac{(\alpha+\gamma)}{6} A^{2}+\frac{\left(\omega+\alpha k^{2}\right)}{2} \frac{A^{2}}{B^{2}}-\frac{\beta}{6} \frac{A^{4}}{B^{2}}=0 .
$$

Solving Eqs. (30) and (31), we obtain

$A= \pm \sqrt{\frac{2\left(\omega+\alpha k^{2}\right)}{\beta}}$

and

$B= \pm \sqrt{\frac{\left(\omega+\alpha k^{2}\right)}{\alpha+\gamma}}$

Substituting these values of $A$ and $B$ into Eq. (28), we obtain

$u(\xi)= \pm \sqrt{\frac{2\left(\omega+\alpha k^{2}\right)}{\beta}} \operatorname{sech}\left\{\left(\sqrt{\frac{\omega+\alpha k^{2}}{\alpha+\gamma}}\right) \xi\right\}$.

Substituting Eq. (34) with the positive coefficient into Eq. (3) and recalling the expressions for $v$ and $\xi$, we obtain the bright or nontopological soliton solution of Eq. (2) as

$\Psi(x, t)=$

$\sqrt{\frac{2\left(\omega+\alpha k^{2}\right)}{\beta}}\left[\operatorname{sech}\left\{\left(\sqrt{\frac{\omega+\alpha k^{2}}{\alpha+\gamma}}\right)(x+2 \alpha k t)\right\}\right] e^{i(-k x+\omega t)}$.

\section{Discussion}

From all of the three methods used here, we obtain the same bright or non-topological 1-soliton solution of the Resonant Nonlinear Schrodinger Equation with Kerr law nonlinearity. Such a soliton will have a bell-shaped profile.

\section{Conclusion}

In this paper, three methods namely (1) the Ansatz method, (2) the Sine-cosine method and (3) the Semi-inverse variation method have been applied in obtaining Bright or Non-topological 1soliton solution of Resonant Nonlinear Schrodinger Equation with Kerr law nonlinearity and the results reveal that these methods are powerful tools for obtaining exact solutions of various types of Nonlinear Evolution Equations (NLEEs) in the studies of science and engineering.

\section{References}

[1] Ekici, M., Zhao, Q., Sonmezoglu, A., Manafian, J. and Mirzaza deh, M., The analytical study of solitons to the nonlinear Schrodinger equation with resonant nonlinearity, Optik - International Journal for Light and Electron Optics https://doi.org/10.1016/j.ijleo.2016.10.098.

[2] Eslami, M., Mirzazadeh, M. and Biswas, A., Soliton solutions of the resonant nonlinear Schrodinger's equation in optical fibers with time dependent coefficients by simplest equation approach, Journal of Modern Optics, 60(19), 2013, $1627 \quad-1636$ https://doi.org/10.1080/09500340.2013.850777.

[3] Guner, O., Bright and dark soliton solution of the nonlinear partial differential equations system, New Trends in Mathematical Sciences, 3(4), 2015, $154-163$.

[4] Mirzazadeh, M., Topological and non-topological soliton solutions of Hamiltonian amplitude equation by He's semi-inverse method 
and ansatz approach, Journal of Egyptian Mathematical Society, 23, 2015, 292 - 296. https://doi.org/10.1016/j.joems.2014.06.005.

[5] Triki, H., Yildrim, A., Hayat, T., Aldossar, O. M. and Biswas, A. Topological and non-topological soliton solutions of the Bertherton equation, Proceedings of the Romanian Academy Series A, vol. 13, No. 2, 2012, $103-108$.

[6] Bekir, A., New exact travelling wave solutions of some complex nonlinear equations, Communications in Nonlinear Science and Numerical Simulations, 14, 2009, $1069 \quad-1077$. https://doi.org/10.1016/j.cnsns.2008.05.007.

[7] Gao, H., Symbolic Computation and New Exact Travelling Solutions for the $(2+1)$ Dimensional Zoomeron Equation, International Journal of Modern Nonlinear Theory and Applications, 3, 2014, 23 -28 .

[8] Jawad, A. J. M., The Sine - Cosine Function Method For The Exact Solutions Of Nonlinear Partial Differential Equations, Ijrras 13(1), Oct. 2012.

[9] Wazwaz, A. M., A Sine - Cosine Method for Handling Nonlinear wave Equations, Mathematical and Computer Modelling, 40, 2004, 499 - 508. https://doi.org/10.1016/j.mcm.2003.12.010.

[10] Zhao, H., Geng, S. and Tang, S. Q., Sine - Cosine Method for a Class of Nonlinear Fourth Order Variant of a generalized CamassaHolm Equation, British Journal of Mathematics and Computer Science 4(11), 2014, $1534 \quad-\quad 1541$. https://doi.org/10.9734/BJMCS/2014/7892.

[11] Biswas, A., Soliton Solutions of the Perturbed Resonant Nonlinear Schrodinger Equation with Full Nonlinearity by Semi-inverse Variational Principle, Quantum Physics Letters 1(2), 2013, 79 - 83.

[12] El - Ganaini, S., Applications of He's Variational principle and the First Integral Method to the Gardner Equation, Applied Mathematical Sciences, 6(86), 2012, 4249 - 4260.

[13] Jabbari, A., Kheiri, H. and Bekir, A., Exact solutions of the coupled Higgs equation and the Maccari system using He's semi - inverse method and $\left(\mathrm{G}^{\wedge^{\prime}} / \mathrm{G}\right)$ - expansion method, Computers and Mathematics with Applications, 62, 2011, $2177 \quad$ - 2186. https://doi.org/10.1016/j.camwa.2011.07.003.

[14] Kheir, H., Jabbari, A., Yildrim, A. and Alomari, A. K., He's semiinverse method for soliton solutions of Boussinesq system, World Journal of Modelling and Simulation, 9 (1), 2013, 3013.

[15] Najafi, M., Arbabi, S. and Najafi, M., He's semi-inverse method for Camassa-Holm equation and simplified modified Camassa-Holm equation, International Journal of Physical Research 1(1), 2013, 1 6. https://doi.org/10.14419/ijpr.v1i1.679. 